\title{
PATIENTS PREFERENCE FOR EXODONTIA VERSUS PRESERVATION IN MALAYSIA
}

Type: Article

Abstract:

A total of 537 dentate adults from nine randomly selected government dental centers in three states in Malaysia were interviewed to assess their preference for either exodontia or preservation of teeth when they experience toothache, or have carious anterior or posterior teeth. The assumptions tested were i) patients prefer exodontia rather than preservation when they have toothache, and ii) patients are more willing to have posterior teeth extracted than anterior teeth for caries. Both these assumptions were rejected. Even though the majority of the subjects preferred preservation (59\%) when having toothache, exodontia was the treatment of choice in a large proportion of subjects $(41 \%)$. Significant differences in preference were found among the various ethnic, educational, income, and age groups. However, when ethnicity was held constant, binary regression indicated that the variations observed were determined by education, income, and age groups and not by ethnicity.

\begin{tabular}{|c|l|}
\hline Author & $\begin{array}{l}\bullet \text { Razak, I. A. } \\
\end{array}$ \\
& $\begin{array}{r}\bullet \text { Jaafar, N. } \\
\text { Jalalludin, R. L. }\end{array}$ \\
\hline Source & Community Dentistry and Oral Epidemiology \\
\hline ISSN & $0301-5661$ \\
\hline DOI & $10.1111 /$ j.1600-0528.1990.tb00037.x \\
\hline Volume (Issue) & $18(3)$ \\
\hline Page & $131-132$ \\
\hline Year & June 1990 \\
\hline
\end{tabular}

Keyword:

Adolescent, adult, age, article, dental surgery, ethnic group, female, human, income, Malaysia, Male, patient attitude, psychological aspect, socioeconomics, tooth extraction, tooth pain, Age Factors, Dental Restoration, Permanent, Educational Status, Ethnic Groups, Middle Age, Patient Acceptance of Health Care

Please Cite As:

RAZAK, I. A., JAAFAR, N., JALALLUDIN, R. L. \& ESA, R. 1990. PATIENTS PREFERENCE FOR EXODONTIA VERSUS PRESERVATION IN MALAYSIA. Community Dentistry and Oral Epidemiology, 18, 131-132. 
URL:

- apps.webofknowledge.com Search by Accession No: A1990DK02200004

- http://www.scopus.com/inward/record.url?eid=2-s2.00025440208\&partnerlD=40\&md5=3baf564167c4309f87f59c13601c4103

- http://onlinelibrary.wiley.com/doi/10.1111/j.1600-

0528.1990.tb00037.x/abstract?systemMessage $=$ Wiley+Online+Library+will+be+d isrupted+on+7+July+from+10\%3A00-12\%3A00+BST+\%2805\%3A0007\%3A00+EDT\%29+for+essential+maintenance

- http://www.ncbi.nlm.nih.gov/pubmed/2350948

- http://ejum.fsktm.um.edu.my/article/541.pdf 